\title{
ENABLING HETEROGENOUS MULTI-SCALE DATABASE FOR EMERGENCY SERVICE FUNCTIONS THROUGH GEOINFORMATION TECHNOLOGIES
}

\author{
V.Bhanumurthy, K. Venugopala Rao, S. Srinivasa Rao, K.Ram Mohan Rao*，P.Satya Chandra，J. Vidhyasagar， P.G.Diwakar, \\ V.K.Dadhwal \\ National Remote Sensing Centre, Indian Space Research Organisation, Hyderabad, India - (bhanumurthy_v, venu_koppaka, ssrao, \\ rao_krm, satyachandra_p, vidhyasagar_j, pgdiwakar, director)@nrsc.gov.in
}

\section{Commission VI, WG VI/4}

KEY WORDS: GIS, Emergency Management, Database, Schema, Emergency Response, NDEM.

\begin{abstract}
:
Geographical Information Science (GIS) is now graduated from traditional desktop system to Internet system. Internet GIS is emerging as one of the most promising technologies for addressing Emergency Management. Web services with different privileges are playing an important role in dissemination of the emergency services to the decision makers. Spatial database is one of the most important components in the successful implementation of Emergency Management. It contains spatial data in the form of raster, vector, linked with non-spatial information. Comprehensive data is required to handle emergency situation in different phases. These database elements comprise core data, hazard specific data, corresponding attribute data, and live data coming from the remote locations. Core data sets are minimum required data including base, thematic, infrastructure layers to handle disasters. Disaster specific information is required to handle a particular disaster situation like flood, cyclone, forest fire, earth quake, land slide, drought. In addition to this Emergency Management require many types of data with spatial and temporal attributes that should be made available to the key players in the right format at right time. The vector database needs to be complemented with required resolution satellite imagery for visualisation and analysis in disaster management. Therefore, the database is interconnected and comprehensive to meet the requirement of an Emergency Management. This kind of integrated, comprehensive and structured database with appropriate information is required to obtain right information at right time for the right people. However, building spatial database for Emergency Management is a challenging task because of the key issues such as availability of data, sharing policies, compatible geospatial standards, data interoperability etc. Therefore, to facilitate using, sharing, and integrating the spatial data, there is a need to define standards to build emergency database systems. These include aspects such as i) data integration procedures namely standard coding scheme, schema, meta data format, spatial format ii) database organisation mechanism covering data management, catalogues, data models iii) database dissemination through a suitable environment, as a standard service for effective service dissemination.
\end{abstract}

National Database for Emergency Management (NDEM) is such a comprehensive database for addressing disasters in India at the national level. This paper explains standards for integrating, organising the multi-scale and multi-source data with effective emergency response using customized user interfaces for NDEM. It presents standard procedure for building comprehensive emergency information systems for enabling emergency specific functions through geospatial technologies.

\section{INTRODUCTION}

Geospatial database has become an important asset in Emergency Management. It plays an important role in coordinating various Emergency Management phases enabling work flows from planning to response and visualisation of situation by the means of satellite and multi-scale vector data supported by ancillary data. However, integration of various types of heterogeneous multi-scale data suitable for handling emergency scenarios is a quite challenging task (Sophie and Pierre, 2007). Emergency Management need a comprehensive structured database for addressing situations such as situational awareness, evacuation, proximity of resources, routing etc. and need to take rapid decisions in quick time (Kailash, 2010). It consists of core, hazard specific and secondary data. Core datasets are the minimum data sets that require handling any disaster. These are generally vector data elements comprising of base, thematic, infrastructure layers. Hazard specific layers are the historic data of various and disaster specific layers for damage assessment, relief, mitigation and recovery operations in the event of a disaster. Raster data is a high resolution satellite data or aerial photography data for complementing vector data for situational awareness and visualisation of the ground scenario. Non-spatial data or secondary data is valuable attribute information for decision making and analysis. Therefore, all these database elements put together in a standardised model become a comprehensive database for addressing Emergency Management. However, these data elements may be of different type, format, scale generated or derived from various sources. Therefore integration of the data into a structured database for query and analysis by a uniform data model, storage structure, access functionality is a critical task for Spatial Database Management Systems (Thomas et al. 1998). This paper presents a various types of database elements required for addressing Emergency Management and integration framework and necessary standards for organisation of multiscale geospatial database.

\footnotetext{
* Corresponding author : rao_krm@nrsc.gov.in
} 


\section{DATABASE}

Emergency Management is a complex phenomenon and require very detailed and structured database for taking decisions (Bartel et al, 2010). However, availability of information is very critical and should be made available to key players for decision making. The success of decision making will largely depends on the availability of information as GIS is data driven system. The essential database elements for Emergency Management framework comprises of Core, Hazard specific and Non-spatial data. Core data is covering base layers, thematic layers, infrastructure layers along with raster data sets. These include data layers such as administrative boundaries, forest boundaries, canal, drainage, land use/land cover, slope, railway stations, river gauze stations etc. Seamless road/rail network along with Point of Interests (PoIs) such as primary health centres, hospitals, schools, shelters, parking areas, bridges, culverts, dams, embankments, barrages, Check Dams, flyovers etc. are also very essential for addressing emergency situations such as emergency trends, demographic patterns, economic profiles, infrastructure status, communication networks, public utilities etc. Hazard specific database is temporal disaster specific database covering flood/cyclone layers; forest fires; Tsunami; Drought; Landslides; Earthquakes and other technological disasters. Non-spatial data provide additional attribute information relevant for necessary query and analysis. So the standardised multi-scale database with core, hazard specific and attribute data is essentially to serve as a national repository of database for emergency/disaster management to assist the disaster managers at various levels in preparedness, relief \& response, damage assessment, and recovery operations (Sha and Xie, 2010 ).

The creation of a robust database is most important task for implementation of Emergency Management objectives. The entire database envisaged can be categorized into Vector, Raster and Attribute data. Vectors are a versatile and frequently used geographic data representation, well-suited for representing features with discrete boundaries such as administrative boundary, canal, forest, rail, road, Point of Interests etc.
Typically, these features are spatially represented as points, lines, or polygons. Raster model also considered for organisation of satellite, aerial data for complementing the vector data sets. A separate data model is used to store and maintain the non-spatial data along with vector data. Figure 1 shows the essential database elements required for addressing Emergency Management.

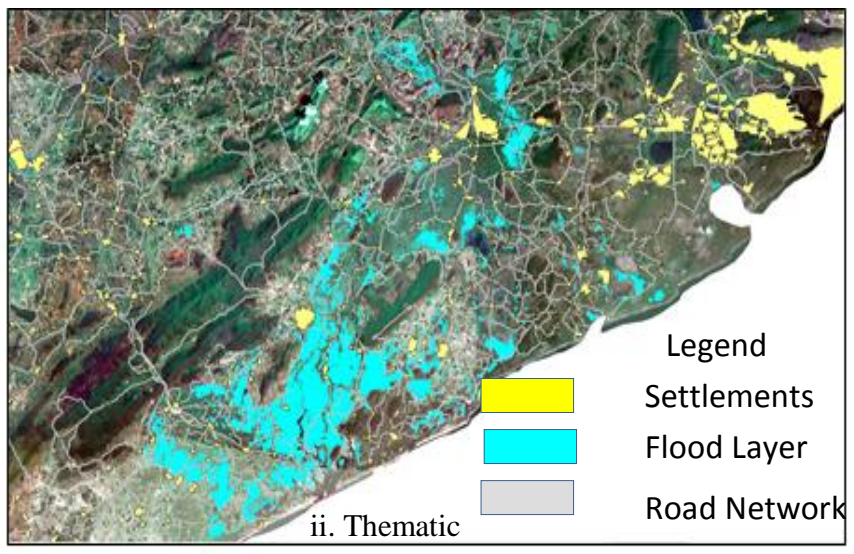

\begin{tabular}{|c|c|c|c|c|c|}
\hline Name & TRU & No_HH & TOT_P & TOT_M & TOT_F \\
\hline Samba & Total & 65385 & 318898 & 169124 & 149774 \\
\hline Samba & Rural & 54718 & 265283 & 139365 & 125918 \\
\hline Samba & Urban & 10667 & 53615 & 29759 & 23856 \\
\hline Samba & Total & 65385 & 318898 & 169124 & 149774 \\
\hline Samba & Rural & 54718 & 265283 & 139365 & 125918 \\
\hline Samba & Urban & 10667 & 53615 & 29759 & 23856 \\
\hline Chani Mar & Rural & 666 & 3216 & 1685 & 1531 \\
\hline Deawan & Rural & 362 & 2059 & 1082 & 977 \\
\hline Kat Walta & Rural & 456 & 2306 & 1196 & 1110 \\
\hline Purmand $\tilde{z}$ & Rural & 250 & 1276 & 662 & 614 \\
\hline Talhar & Rural & 18 & 104 & 47 & 57 \\
\hline Padal & Rural & 40 & 287 & 146 & 141 \\
\hline Rajoul & Rural & 36 & 204 & 110 & 94 \\
\hline Khathar Bı & Rural & 39 & 217 & 119 & 98 \\
\hline Kumi & Rural & 36 & 212 & 107 & 105 \\
\hline Mahur Grá & Rural & 169 & 848 & 436 & 412 \\
\hline Dergarh & Rural & 23 & 123 & 64 & 59 \\
\hline ـم متصمصصما & لمعسم & 7م:1 & $=2 \varepsilon_{1}$ & בحمح - 1 & لحمح r r r \\
\hline
\end{tabular}

iii. Non-Spatial

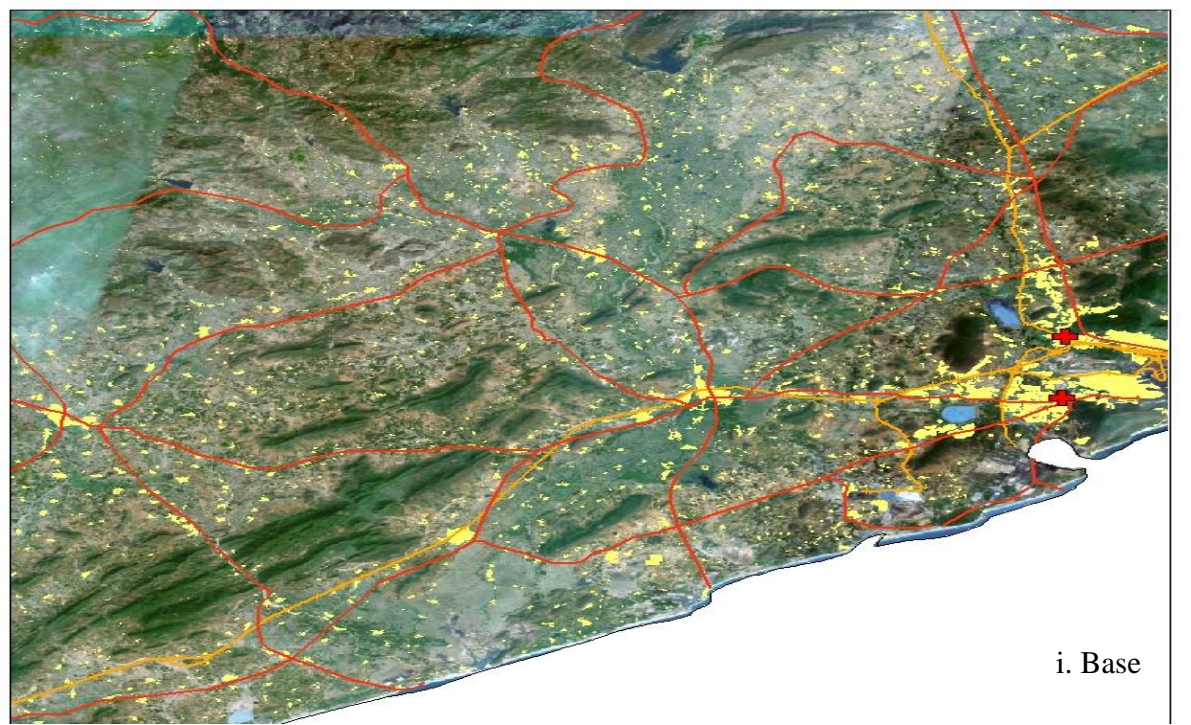

Figure 1. Essential database elements (i.Base ii. Thematic and iii. Non-spatial)

\section{Legend}

Sea Port

Air Port

Hospital

Point of Interest

Road Network

Rail Network

Settlement Area

Administrative Boundary 


\section{INTEGRATION FRAMEWORK}

Achieving the comprehensive spatial database is the ultimate objective of the country for holistic approach of combating disasters. Many organisations have achieved the generation of the specialised database in their disciplines. But it is very much tough situation for any individual organisation to generate a national database required for addressing a comprehensive disaster management. Sharing of the database for effective disaster management is the need of the hour. Spatial Data Infrastructure (SDI), clearing houses are already embarked on the importance of spatial data access and sharing without regeneration of the spatial database. Now, it is the time to integrate and analyse the data using spatial analysis, data mining, pattern recognition etc. for strengthening Emergency Management systems. However, effective integration and interoperability of multi-source, multi-scale data is more important for any country. It provides a solid foundation for achieving a comprehensive platform for decision making.

\subsection{Integration of multi-scale data}

Spatial data integration is the process of unifying different sets of available data within GIS platform are made compatible with each other. Many datasets are generated in the native formats, projections, and even their own interests of attributes. It is also observed that most of the data is generated in a different scale as well. For example, a road network of a particular state is digitised at 1:50,000 scale. The same road network of an individual city in that State is generated at 1:5,000 scale. In both the cases data need to make compatible with each other, and must be used appropriately for a network routing in the case of an emergency. The other major problem in integration is relating incompatibility of the spatial attributes for which data is recorded. For performing network analysis, the road network musts have the network parameters which may not be available in the attributes at State level. In this case, these data sets need to be compared and updated with the data sets having the relevant network parameters and generated in improved scale using map matching algorithms (Anurag et al, 2010). Data integration includes following aspects (figure 2).

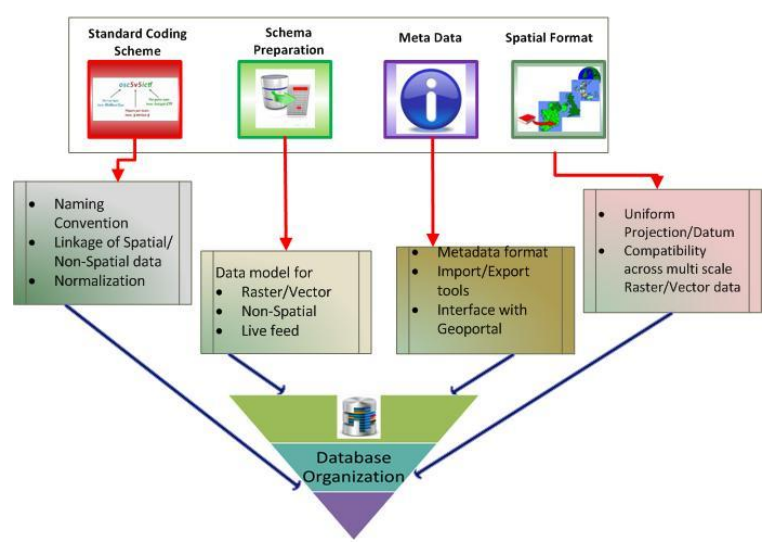

Figure 2. Data integration procedures

Standard coding scheme - File naming is designed to provide unique reference for each layer that is organized in the database.
Schema preparation - The structure or design of database such as features, table, view, index, stored procedure etc. are defined in the schema.

Meta data - From a data management prospective, Meta data is important to maintain in database with incremental versions.

Spatial format - Spatial framework is the most critical aspect for achieving seamlessness and compatibility of the multi-scale spatial database. The framework is a combination of datum, projection and bounding limits definition that allows easy and accurate transformation and visualization of the spatial information in the repository.

Integration procedures help to standardise the format, naming conventions and compatibility etc., to enable effective database management. The datasets pooled from the various sources are integrated through the above mentioned procedures for the effective database organisation.

\subsection{Organisation of database}

Emergency database is a unique database of its type, having comprehensive layers covering base, thematic, infrastructure layers. The database also complemented with satellite imagery for visualisation and analysis. Detailed ancillary data is also linked with spatial information for extensive analysis. In addition to this, it has disaster specific layers. Therefore, it is an interconnected database to meet the visualisation of database in the case of disasters and for customisation of emergency service functions for addressing Emergency Management in the country. Organisation of multi-scale database includes three phases (figure 3).

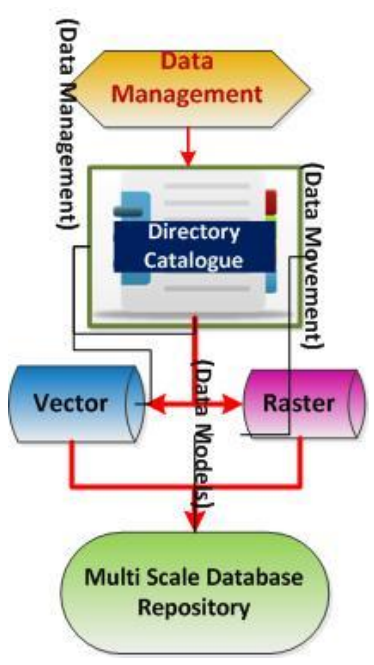

Figure 3. Database Organisation phases

Data management - It describes the procedures for organizing and controlling multi-scale database pooled from several agencies from multiple locations, this data is collected and stored in a master database and subsequently various data management operations are performed to re-organize the database as per the extent of the study area. To organize the database, the raster/vector database is undergone with various functions for seamless database generation in various scales. 
Directory catalogue - It is compilation of information about database layers. Once the identified database elements are finalized with predefined data management operations, a standardized database catalogue is worked out to meet the organization as per the objectives at different scales. All these datasets are organized in a structured way in directory catalogue in the central storage for secure storage and easy retrieval.

Database repository -Ensuring that all geospatial database elements are stored in the proper location within a standardized directory structure in central storage, enables an efficient workflow, reduces ambiguity, for easier relocation of data or products. It also ensures a smooth incident transition between GIS database organisation, serving and the data archival process.

\subsection{Hosting of emergency services}

This database repository is the input for database hosting and further services to users and decision makers. Dissemination of data is an equally important aspect. In fact, the main aim of the data is to serve the multi scale geospatial data to the various users for decision making to address the emergency situations arising out of natural or man-made disasters. However, deployment and dissemination of the data needs to be provided through a secure and stable network using OGC Standards (OGC, 2006). Thus, the proper hosting of data along with secure measures must be the strength of the information system. The generic data flow of the applications includes 3 tier architecture (figure 4). Layer 1 is consistent storage of databases stored in central storage in vector / raster/ Non-spatial formats. Layer 2 is an application server for accessing the database layers for serving to end users as rendered products. Layer 3 is a user interface for providing the access to the geoservices to the end user / decision maker.

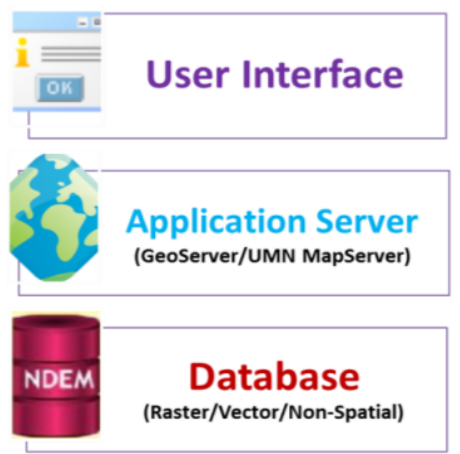

Figure 4. Application architecture

As apparent from the essential elements for Emergency Management, the database is highly complex with different formats and numerous sources involved. Much of the data is spatial in nature especially lot of high resolution satellite imagery ranging from $5 \mathrm{mts}$ to a sub meter resolution, digital elevation data, aerial data etc. The amount of associated data is massive and since some of it is also temporal in nature, the size of the dataset will increase exponentially with the passage of time. In these data sets, core data sets are commonly required to handle most of the emergency situations. Hazard specific data sets are required to handle a particular emergency situation, such as information on health facilities, flood hazard layers, Landslide Hazard layers etc. In all these scenarios, visualisation of raster/ vector data of the incident study area along with transportation network connectivity, various point of interests such as hospitals, clinics, shelters, schools, parking area, petrol pumps etc. is essential for identifying resources in the case of an emergency. The vector data sets at individual scales are organised as shape files for further integration with a higher level data model. Similarly, raster data sets are organized at file level. The entire data sets are served as geospatial web service.

\section{NATIONAL DATABASE FOR EMERGENCY MANAGEMENT}

National Database for Emergency Management (NDEM) is a GIS based national database to support disaster/ Emergency Management in India. Presently, it covers natural disasters such as flood, cyclone, forest fire, earthquake, landslide (figure 5). This database, which will leverage much on the aerospace data, will have core data, hazard-specific data, and dynamic data in spatial as well as non-spatial forms. The NDEM will contain datasets at different scales. As the different datasets ingesting into the NDEM are generated by different central/state organizations/agencies, the implementation of NDEM is planned as a coordinated effort.

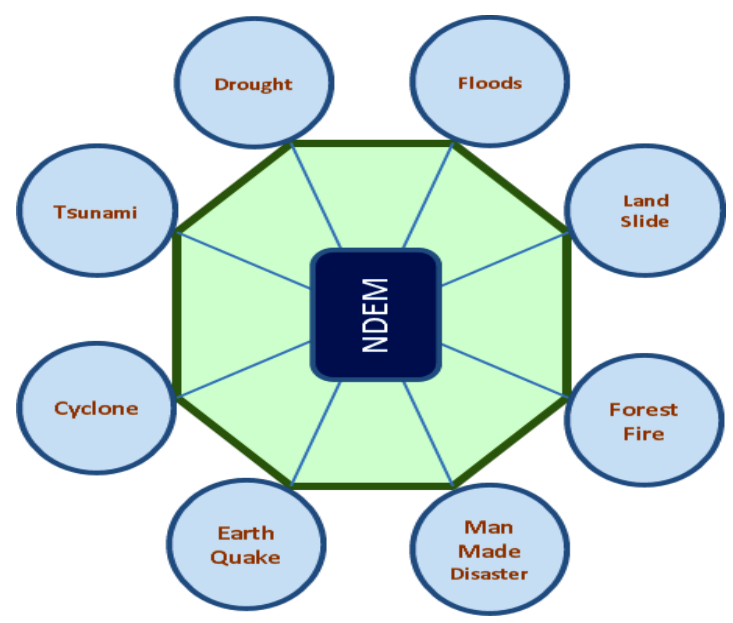

Figure 5. NDEM database for natural disasters

The databases are organized and integrated through a sophisticated model as a central database repository for serving the databases as Web Map Service (WMS) to NDEM internal users and for the further development of Decision Support System tools in the form of customized graphical user interfaces. The main objectives of NDEM are organization of multi-scale geospatial database(s) covering National Database of entire India at 1:50,000 scale, multi-hazard prone districts at $1: 10,000$ scale, and mega cities at 1:2,000 scale and development of customised Decision support (DSS) tools for addressing disaster/Emergency Management in the country.

\subsection{Scope of the database}

NDEM envisages having a number of spatial layers relating to core data sets at 1:50000 scale for entire country which is commonly required to handle most of the emergency situations. At 1:10000 scale, spatial layers namely urban ward boundaries, villages, roads, forest, transport nodes, urban land use, urban plans with detailed attribute information is essential for addressing hazard zonation, routing, natural resources, 
hazardous industries, resource inventories, etc. The data at 1:2000 scale is identified for 5 mega cities to help Emergency Management. In addition to the above stated vector data sets, raster data sets containing high resolution satellite imagery, aerial photographs are also integrated with for visualisation and decision making. Database available with ISRO (generated under various national projects) are made available to NDEM. As the different datasets ingested into the NDEM are generated by different organizations/agencies, the implementation of NDEM is planned as a coordinated inter-ministerial effort. In this regard nodal officers from the Central / State Departments concerned for providing the database available with them for NDEM Project. Attempts were made through identified nodal officers to obtain the detailed inventory of the available information with the respective departments in the specified format. Overall, the database contains core data, disaster specific data along with non-spatial data which will be integrated as a multi-scale geospatial data for addressing Emergency Management.

These data sets are organized using integration strategies for database organisation and translation to NDEM framework (figure 6). Further, any other modification required for the existing datasets for integration has to be undertaken. Further it was decided to initiate the work with the readily available data sets so that the envisaged NDEM is realized and made operational to certain extent. During the course of execution of NDEM project some suggestions were obtained, resulting in some changes in the scope of NDEM project. In the changed scenario the database is arranged for:

- $\quad$ Entire India (state wise) at 1:50,000 scale

- Total 350 districts at 1:10,000 Scale

- $\quad$ Large scale urban specific data for 5 Mega Cities at 1:2,000 scale

The databases are focussed on the timely provision of necessary geospatial information to all the stake holders in emergency /disaster management at various levels of disasters.
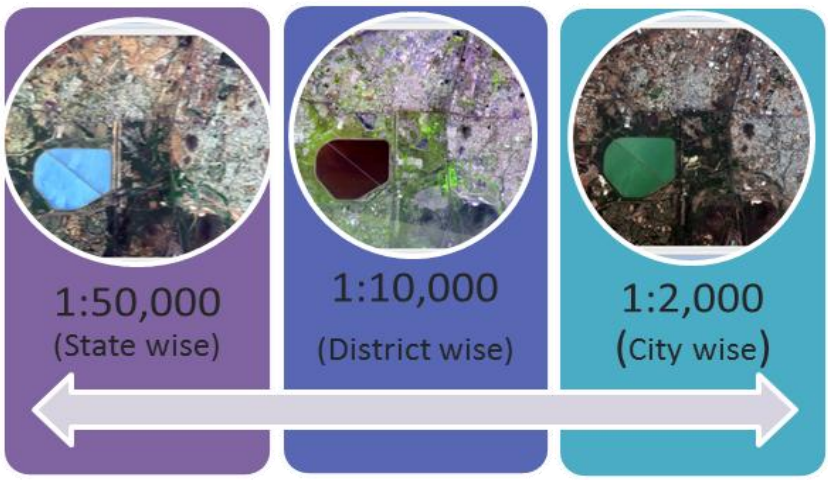

Figure 6. NDEM multiscale database

For realising this, a comprehensive database is organized for meeting the natural and technological disasters with the envisaged database. With reference to the database objectives, the precise data layers from the envisaged list are categorised and organised in the form of standardised master database list including vector and raster in a spatial format. These layers are standardised as per NNRMS content standard and the attributes are generated as per the NNRMS LUT references (NNRMS Standard Committee, 2005).

\subsection{Database services}

It is proposed to organise the database at state level for entire country. Each state will have a geospatial at 1:50000, district wise datasets at 1:1000 scale and large scale urban information at 1:2000 scale. All these data sets are integrated along with corresponding non-spatial information for query and retrieval purpose. The vector data sets at individual scales are organised as shape files for further integration with a higher level data model. Similarly, raster data sets are organized at file level. The entire data sets are served as geospatial web service through NDEM VPN Service (figure 7). The database services for all States are provided as WMS service and some of the services are shown in figure 8.

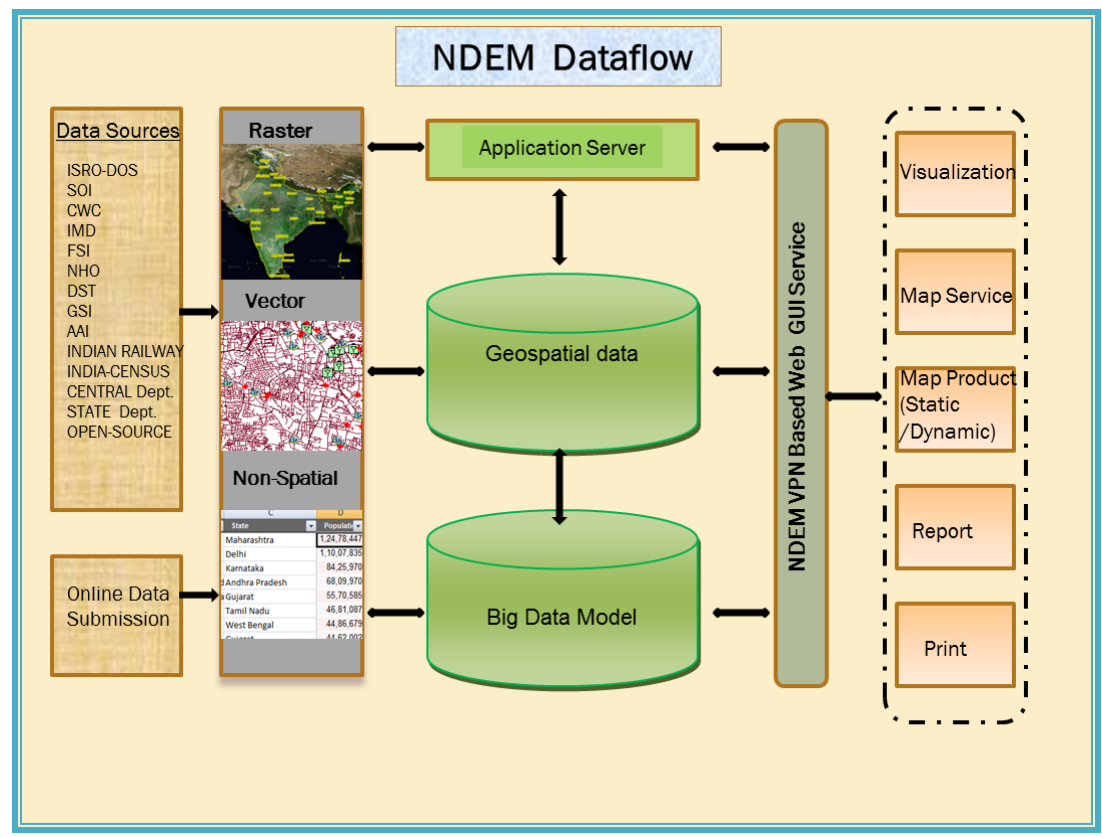

Figure 7. NDEM data flow 


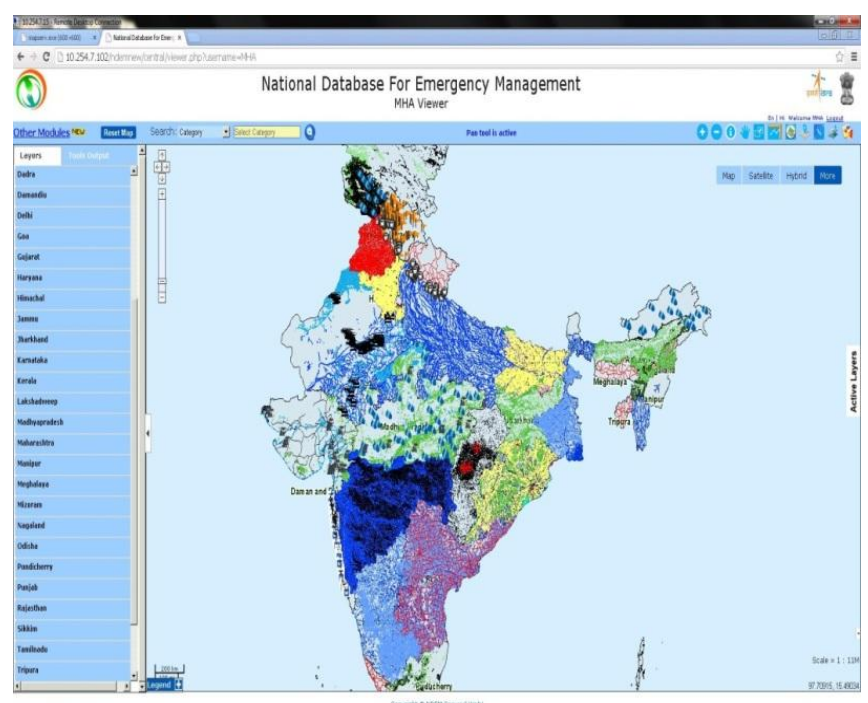

i) State wise database service of entire India

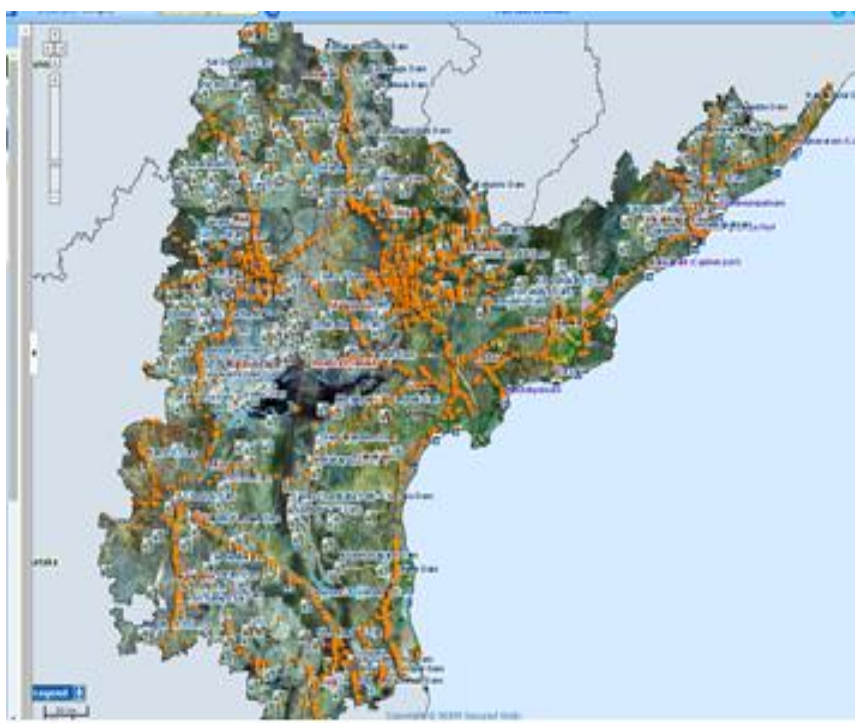

iii) State wise database service of Andhra Pradesh

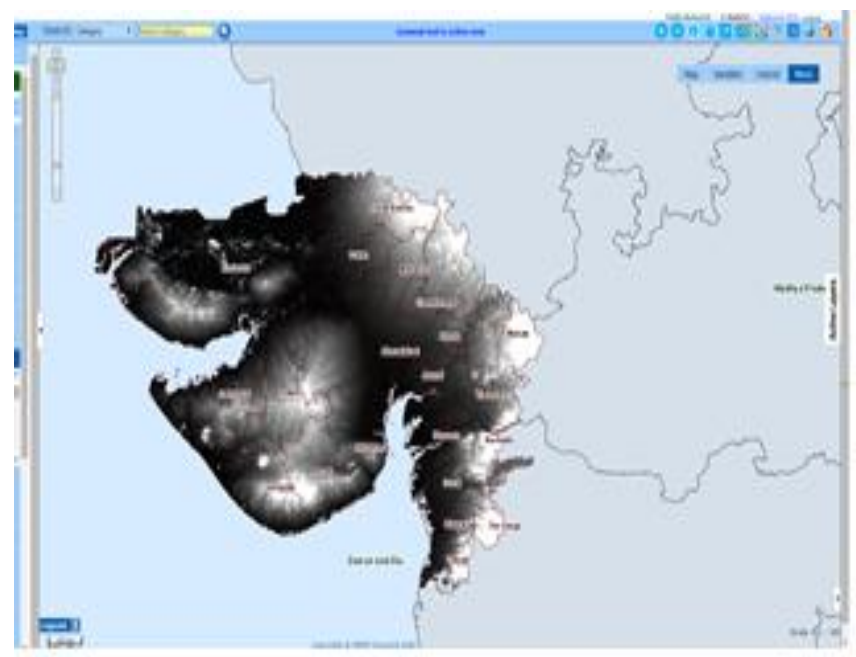

v) DEM as a service of Gujarat State

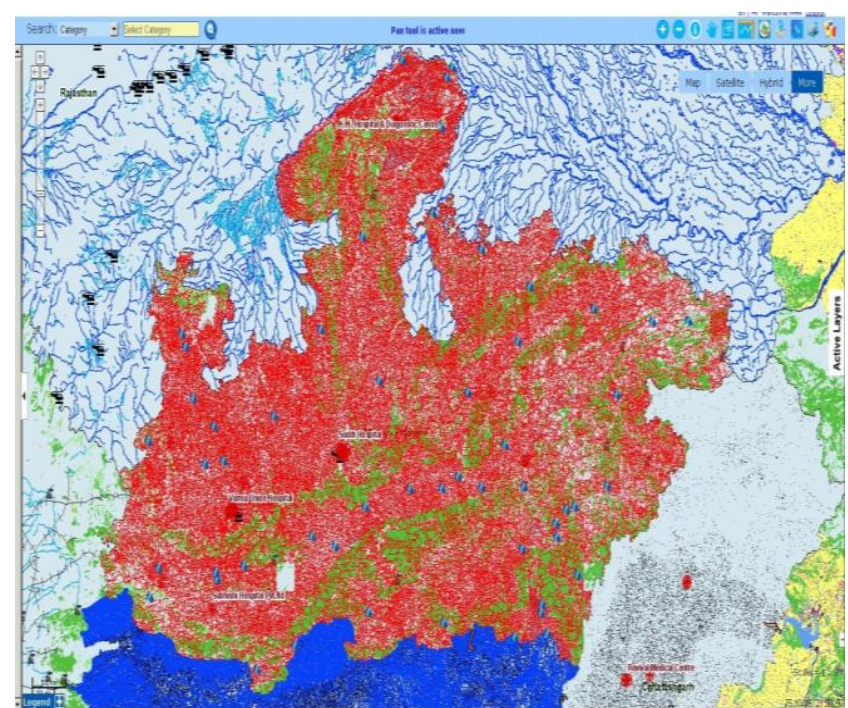

ii) State wise database core data services

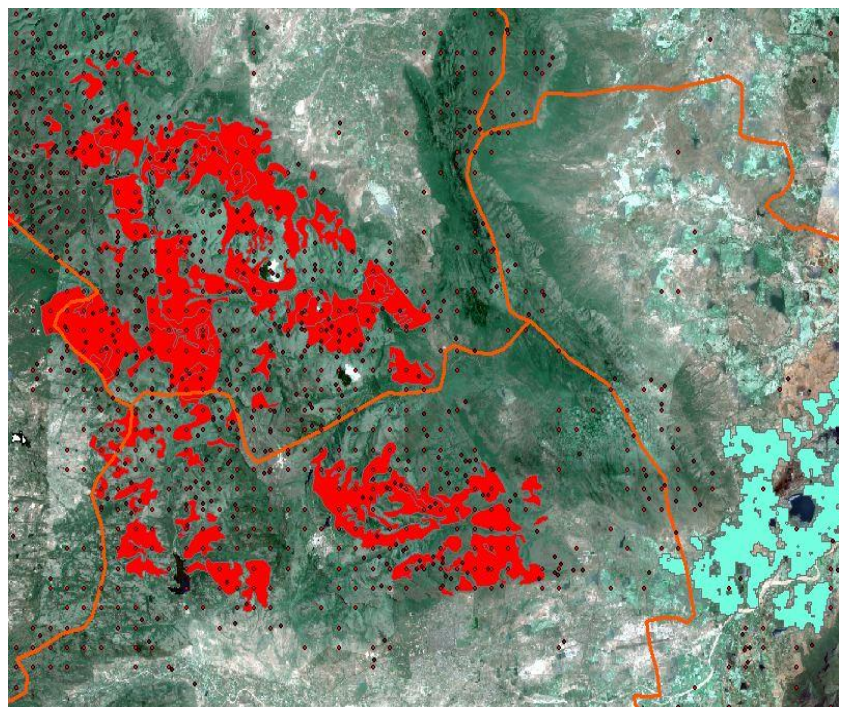

iv) Event wise disaster specific data

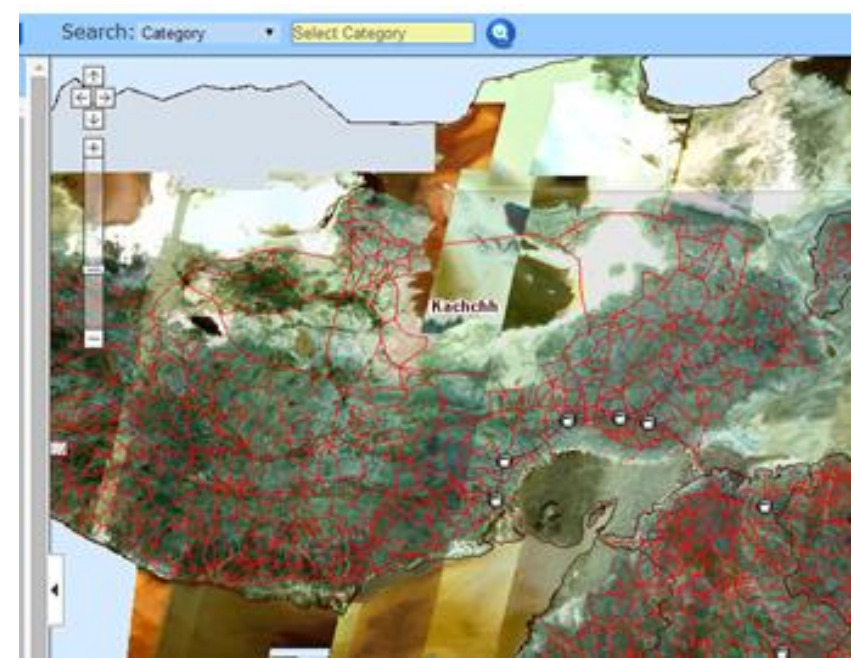

vi) Core data service of Gujarat State

Figure 8. Multi-scale database Services through NDEM portal 
The entire database of NDEM is organized in vector and raster formats. The vector files are organized in flat file model in shape file format. This component enables the shape file to provide geometric features along with attributes to the corresponding geometry. Similarly raster data sets are organized in tiff format for further visualisation. NDEM database is hosted into a VPN environment for providing web services to the States and the internal users for further customization of DSS tools. The technologies being used in the hosting are i)Geoserver for vector hosting; ii)UMN MapServer for raster hosting.

The objective of the NDEM database organisation is to provide Organization of multi-scale geospatial database(s) covering National Database of entire India at 1:50,000 scale, multihazard prone districts at 1:10,000 scale, and mega cities at 1:2,000 scale. These databases are provided WMS/WFS service to the various users for data dissemination and visualisation.

\subsection{System Architecture}

The NDEM Database System is customised using geographic databases, servers of interactive maps that generate services on maps to provide Web Map Service (WMS)/ Web Feature Service (WFS). The database catalogue is also customized as per the NDEM database structure so that users can access the database in an easy to understand format for the visualisation and query. Figure 9 shows the system architecture. The database is stored in a central storage and hosted using WebGIS tools for disseminating the database as a WMS accessible to the users. Similarly, the database is used to customize the Decision Support Tools for decision making. Java application server is used to customize the application user interface and DSS tools. Figure 10 shows the optimal routing deployed using the road network for a disaster event.

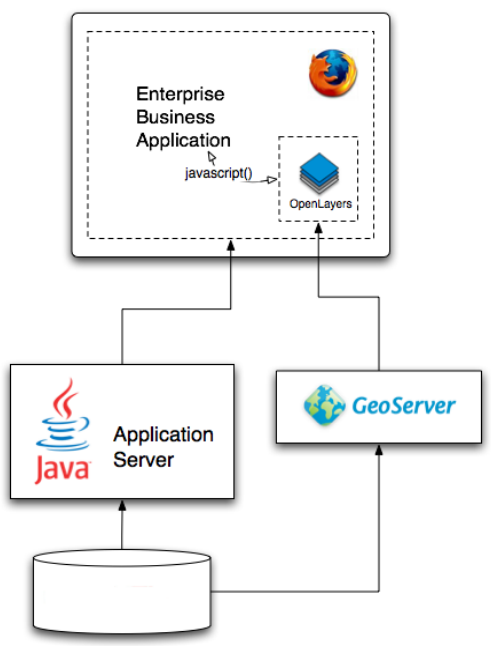

Figure 9. NDEM Service Architecture

\section{CONCLUSION}

Database organisation is the one of the most important component in the successful implementation of NDEM. It contains spatial data in the form of raster, vector, linked with non-spatial information. Specific data sets are required to handle disaster situation in different phases. These database elements comprise core data, hazard specific data, corresponding attribute data, and live data coming from the remote locations. Core data sets are minimum required data including base, thematic, infrastructure layers to handle disasters. Disaster specific information is required to handle a particular disaster situation like flood, cyclone, forest fire, earth quake, land slide, drought.

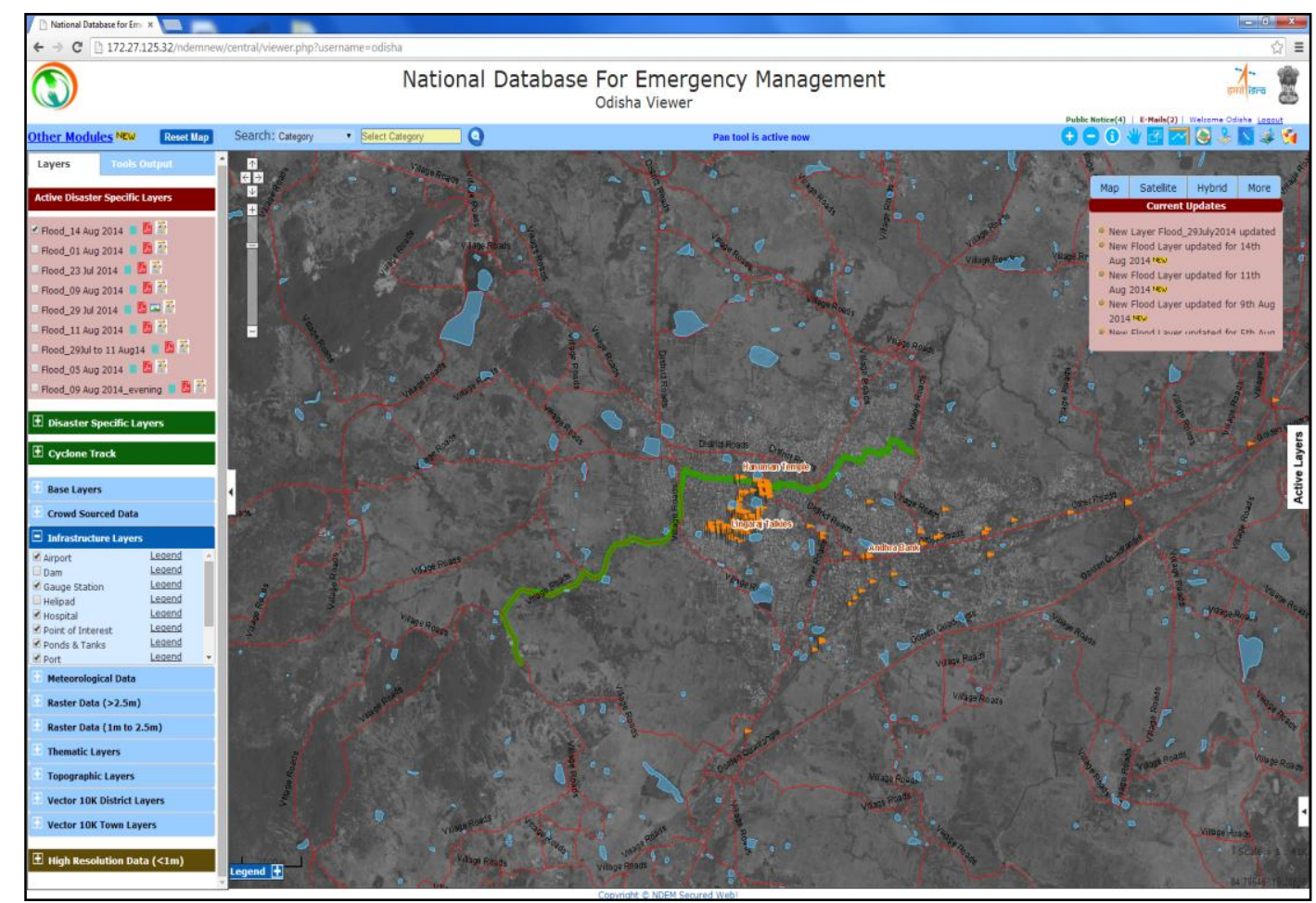

Figure 10. Spatial analysis 
The vector database needs to be complemented with required resolution satellite imagery for visualisation and analysis in disaster management. Therefore, to facilitate using, sharing, and integrating the spatial data, there is a need to define standards to organise spatial data. These include aspects such as data integration procedures namely standard coding scheme, schema, meta data format, spatial format, database organisation mechanism covering data management, catalogues, data models and database dissemination through a suitable environment like web, as a standard service for effective service dissemination.

NDEM is a comprehensive national database for addressing emergency Management in the country. For each State geospatial database is integrated as a combination of vector and raster along with attribute information. At 1:50,000 scale, database for 35 States / UTs have been organized and served through NDEM DMS VPN connectivity. The database comprises of i) Base ii) Thematic iv) Infrastructure v) Disaster Specific layers and v) Satellite image data. The database organisation for each state is completed as envisaged in the project. However, replacement of datasets with new data sets in regular intervals if any / value-addition to data layers is a continuous process in the NDEM Database. This database has been hosted and disseminated to NDEM users as service. The NDEM portal is powered with State wise seamless database for individual State/UT with utility and DSS tools for visualisation, decision making, and report generation.

\section{ACKNOWLEDGEMENTS}

The authors are thankful to Dr. Vinod M Bothale, Dr M V Ravi Kumar, Mrs.P.Sri Lakshmi, Mrs Aneela Korrapati, Mr. Brajesh Kumar, Mohd. Kamaludeen, Ms. Neha Urkude, Mrs. Priya Prabhakar, Dr. Murthy Remilla, Mr VV Sarath Kumar and Mr Rakesh Kumar Sharma for suggestions, encouragement and support. The work has been carried out under the NDEM project, National Remote Sensing Centre, Indian Space Research Organisation, Government of India.

\section{REFERENCES}

Anurag Verma, K. Naveen Kumar, K. Ram Mohan Rao. 2011. Evaluation of Map matching Algorithms for Multi Scale Databases. LAP Lambert Academic Publishing. ISBN-13: 9783-8473-0963-5.

Bartel, V, Murray D W, Starr T .2010. Information Systems for Emergency Management. Advances in Management Information Systems. Armonk NY. ISBN 978-0-7656-2134-4.

Goodchild M F., Haining R., Wise S., and 12 others.1992. Integrating GIS and spatial data analysis : problems and possibilities. International Journal of Geographic Information Systems. Vol.6. No.5. pp. 407-423.

Thomas D., Christine P., Stefano S. 1998. On spatial database integration. International Journal of Geographical Information Science, Vol.12 No.4, pp. 335-352. DOI: 10.1080/136588198241824.

Kailash G. 2010. Design of district emergency operations centres, and the case study of Indian Oil Corporation Jaipur depot Explosion. International Journal of Emergency Management, Vol. 7, Nos. 3/4, 2010.

NNRMS STANDARDS COMMITTEE. 2005. 'NNRMS Standards: A national standards for EO images, thematic, cartographic maps, GIS databases and spatial outputs'. ISRO. Document Control Number: ISRO:NNRMS:TR 112:2005.

OGC. 2006. Open GIS Web Map Server Implementation Specification. Open Geospatial Consortium. Reference number: OGC 06-042. Version: 1.3.0.

Sha Zongyao, Xie Yichun. 2010.Design of Service-Oriented Architecture for Spatial Data Integration and Its Application in Building Web-based GIS Systems. Geo-spatial Information Science. Vol.13 Issue. 1. pp:8-15

DOI 10.1007/s11806-010-0163-7

Sophie Sauvagnargues L, Pierre Alain A. 2007. Using GIS for emergency management: a case study during the 2002 and 2003 flooding in south-east France. International Journal of Emergency Management, Vol. 4, No. 4 pp. 682-703 